\title{
Genome-wide identification and analyses of the $A H L$ gene family in cotton (Gossypium)
}

Lanjie Zhao ${ }^{1 \dagger}$, Youjun $\mathrm{Lü}^{1,2,3+}$, Wei Chen ${ }^{1}$, Jinbo Yao ${ }^{1}$, Yan $\mathrm{Li}^{1}$, Qiulin $\mathrm{Li}^{1}$, Jingwen Pan ${ }^{1}$, Shengtao Fang ${ }^{1}$, Jie Sun ${ }^{3^{*}}$ and Yongshan Zhang ${ }^{1^{*}}$

\begin{abstract}
Background: Members of the AT-HOOK MOTIF CONTAINING NUCLEAR LOCALIZED (AHL) family are involved in various plant biological processes via protein-DNA and protein-protein interaction. However, no the systematic identification and analysis of AHL gene family have been reported in cotton.

Results: To investigate the potential functions of AHLs in cotton, genome-wide identification, expressions and structure analysis of the AHL gene family were performed in this study. 48, 51 and 99 AHL genes were identified from the G.raimondii, G.arboreum and G.hirsutum genome, respectively. Phylogenetic analysis revealed that the AHLS in cotton evolved into 2 clades, Clade-A with 4-5 introns and Clade-B with intronless (excluding AHL20-2). Based on the composition of the AT-hook motif(s) and PPC/DUF 296 domain, AHL proteins were classified into three types (Type-I/-II/-III), with Type-I AHLs forming Clade-B, and the other two types together diversifying in Clade-A. The detection of synteny and collinearity showed that the AHLs expanded with the specific WGD in cotton, and the sequence structure of AHL20-2 showed the tendency of increasing intron in three different Gossypium spp. The ratios of non-synonymous (Ka) and synonymous (Ks) substitution rates of orthologous gene pairs revealed that the AHL genes of G.hirsutum had undergone through various selection pressures, purifying selection mainly in Asubgenome and positive selection mainly in D-subgenome. Examination of their expression patterns showed most of AHLS of Clade-B expressed predominantly in stem, while those of Clade-A in ovules, suggesting that the AHLs within each clade shared similar expression patterns with each other. qRT-PCR analysis further confirmed that some GhAHLs higher expression in stems and ovules.
\end{abstract}

Conclusion: In this study, 48, 51 and 99 AHL genes were identified from three cotton genomes respectively. AHLS in cotton were classified into two clades by phylogenetic relationship and three types based on the composition of motif and domain. The AHLs expanded with segmental duplication, not tandem duplication. The expression profiles of GhAHLs revealed abundant differences in expression levels in various tissues and at different stages of ovules development. Our study provided significant insights into the potential functions of AHLs in regulating the growth and development in cotton.

Keywords: Cotton, AHL family, AT-hook motif, Phylogenetics, Synteny, Expression profile, Ka/Ks

\footnotetext{
* Correspondence: Sunjiezh@vip.sina.com; 13938698299@163.com

'Lanjie Zhao and Youjun Lü contributed equally to this work.

${ }^{3}$ Shihezi University, Shihezi 832003, Xinjiang, China

'State Key Laboratory of Cotton Biology, Institute of Cotton Research of

CAAS, Anyang 455000, Henan, China

Full list of author information is available at the end of the article
}

(c) The Author(s). 2020 Open Access This article is distributed under the terms of the Creative Commons Attribution 4.0 International License (http://creativecommons.org/licenses/by/4.0/), which permits unrestricted use, distribution, and reproduction in any medium, provided you give appropriate credit to the original author(s) and the source, provide a link to the Creative Commons license, and indicate if changes were made. The Creative Commons Public Domain Dedication waiver (http://creativecommons.org/publicdomain/zero/1.0/) applies to the data made available in this article, unless otherwise stated. 


\section{Background}

The AT-Hook Motif Containing Nuclear Localized (AHL) gene family, a conserved transcription factor, has been found in all sequenced plant species [1]. The $A H L$ proteins contain one or two AT-hook(s) and the Plant and Prokaryote Conserved (PPC/DUF296) domain, altering chromatin structure and regulate gene expressions [1-3]. AT-hook motif contains conserved Arg-Gly-Arg motif(s) binding to AT-rich DNA region. PPC domains contain 120 amino acids, sharing the same secondary or tertiary structure with seven $\beta$-sheets partially surrounding a single $\alpha$-helix in a wide range of organisms ranging from prokaryotes to higher plants. The hydrophobic region at the C-terminal is essential to nuclear localization and interaction with each other or themselves $[1,2]$. The $A H L$ gene family regulates plant growth and development by forming DNA-protein and protein-protein homo-/hetero-trimeric complex [3, 4].

Phylogenetic analyses showed that $A H L$ gene in land plants emerged in embryophytes and further diverged into two monophyletic clades predating the divergence of mosses from the rest of the land plants [1,3]. The protein sequences of the PPC domain share unique characteristics within each of the two AHL phylogenetic clades. AT-hook motifs can be divided into two types. Based on type and number of the AT-hook motif(s) and the PPC domain, the AHL proteins can be further classified into three types [3]. In angiosperms, Clade-A AHLs expanded into five subfamilies; while, the ones in Clade$B$ expanded into four subfamilies [1].

The high conservation in molecule organization and evolution suggests a vital function of the $A H L$ gene family in regulation of plant growth and development. Several studies have shown that the $A H L$ gene family plays an important role in regulating the elongation of hypocotyl. In A. thaliana, some members of $A H L$ gene family, such as AHL6, AHL15, AHL22, AHL29 and AHL27, inhibits redundantly the elongation of hypocotyl by repressing the genes associated with auxin signaling [4-7]. AHL gene is related to floral transition and reproductive development of plants [8-12]. Overexpression of GIANT KILLER (GIK), which encodes for an $A H L$ protein, leads to severe reproductive defects and down-regulation of genes involved in patterning and differentiation of reproductive floral organs [13]. AtAHL22 delays flowering by acting as a chromatin remodeling factor that modifies the architecture of chromatin region of the FT gene by modulating both histone $\mathrm{H} 3$ acetylation and methylation [14, 15]. The AHL gene family also shows the instrumental role in maintaining hormones homeostasis and regulation of defense response in plants [16-19]. Studies have shown that there are $20 \mathrm{AHL}$ genes in the rice genome data, which have three different expression patterns [19, 20]. All of the OsAHL genes might be functionally expressed genes with 3 distinct expression patterns [20]. Among them, in rice plants during both seedling and panicle development stages, overexpression of OsAHL1 enhanced multiple stress tolerances, this gene could greatly improve drought resistance of rice plants [19].

Some studies on $A H L$ family genes in plants are mainly focused on the model plant, $A$. thaliana and rice. Recently, Genome-wide, expression profiling, and network analysis of AT-hook gene family in maize will help in the further understanding the role of the this gene family in these this cereal crops [21]. Cotton is one of the most important fiber crop and provides amounts of natural fiber used for textile industry worldwide. Overexpression of GhAT1, the only reported $A H L$ in cotton so far, facilitate the specification of fiber cells by repressing the activity of the lipid transfer protein gene FSltp4 [22]. The AHL gene family in cotton remains a mystery to be solved. The completion of genome sequencing of cotton allows us for comprehensive identification and analysis of gene family in cotton [23-27]. Here, AHLs gene family from three cotton species genome datas were identified by bioinformatics methods, the gene structure features, chromosomal locations, phylogenetic relationships, synteny and expression profiles were illustrated to highlight the potential functional diversity. This study will enhance our understanding of the $A H L$ gene family and providing insight into the potential functional diversity of $A H L$ genes of Gossypium.

\section{Results}

\section{Identification and features of $A H L$ genes in cotton}

To identify the $A H L$ genes, the Blastp and Hmmer search program (HMMER3.0 package) was performed against the protein databases using the AtAHL protein sequences as queries. The candidate $A H L$ genes were confirmed using PROSITE and InterProscan 65.0 software to search for the PPC and the AT-hook motifs. Finally, 12, 15, 21, 48, 51, 99 AHL genes were obtained from Physcomitrella patens ( $P$. patens), Vitis vinifera (V. vinifera), Theobroma cacao (T. cacao), Gossypium raimondii (G. raimondii), Gossypium arboreum (G. arboreum), Gossypium hirsutum (G. hirsutum), respectively. The properties of identified AHLs in cotton were also analyzed by ExPASy (https://web.expasy.org/com pute_pi/). The gene lengths of $A H L$ genes in G.raimondii ranged from $684 \mathrm{bp}$ to $8394 \mathrm{bp}$, which encoding polypeptides from 227 to 396 amino acid with predicted molecular weights ranging from $22.75 \mathrm{kD}$ to $41.38 \mathrm{kDa}$. The theoretical pI ranged from 5.35 to 10.68 with charge from -4 to 18 (Table 1). The AHL genes in G.arboreum and G.hirsutum differed greatly in length (641-10,972 bp), isoelectric point (5.3-10.6), molecular weight (17.22-45.29 kDa) and charge (-5-19) (Additional files 1, 2).

\section{Phylogenetic analysis and gene structures of $A H L$ genes} To elucidate the evolutionary relationship of the $A H L$ gene family in Gossypium, the maximum-likelihood phylogenetic 
Table 1 Information of the AHL genes in G. raimondii

\begin{tabular}{|c|c|c|c|c|c|c|c|c|}
\hline Gene name & Sequence ID & Gene (bp) & CDS (bp) & Protein(aa) & Intron & $\mathrm{MW}^{\mathrm{a}}(\mathrm{kDa})$ & $\mathrm{pl}^{\mathrm{b}}$ & Charge \\
\hline GrAHL1-1 & Gorai.003G167100.1 & 2846 & 984 & 327 & 4 & 33.734 & 9.912 & 8.5 \\
\hline GrAHL1-2 & Gorai.004G203700.1 & 4309 & 1026 & 341 & 4 & 35.305 & 9.994 & 8.5 \\
\hline GrAHL1-3 & Gorai.007G021700.1 & 3420 & 984 & 327 & 4 & 33.742 & 10.038 & 9.5 \\
\hline GrAHL3 & Gorai.008G283600.1 & 3507 & 1008 & 335 & 4 & 35.308 & 8.45 & 6.5 \\
\hline GrAHL5-1 & Gorai.004G211500.1 & 3767 & 1026 & 341 & 4 & 35.485 & 10.638 & 18 \\
\hline GrAHL5-2 & Gorai.008G246700.1 & 4352 & 1023 & 340 & 4 & 35.256 & 10.307 & 16 \\
\hline GrAHL7-1 & Gorai.004G161300.1 & 3148 & 1011 & 336 & 4 & 34.954 & 9.402 & 6 \\
\hline GrAHL7-2 & Gorai.007G091400.1 & 4226 & 996 & 331 & 4 & 34.149 & 9.686 & 5.5 \\
\hline GrAHL9-1 & Gorai.004G158000.1 & 3982 & 1023 & 340 & 4 & 35.007 & 9.669 & 10 \\
\hline GrAHL9-2 & Gorai.008G122100.1 & 2887 & 990 & 329 & 4 & 34.067 & 10.453 & 14 \\
\hline GrAHL9-3 & Gorai.007G098600.1 & 4773 & 1023 & 340 & 4 & 34.978 & 10.486 & 14 \\
\hline GrAHL10 & Gorai.002G112700.1 & 6764 & 1095 & 364 & 4 & 37.03 & 10.27 & 14 \\
\hline GrAHL13-1 & Gorai.004G186000.1 & 4606 & 1191 & 396 & 4 & 41.381 & 10.057 & 16 \\
\hline GrAHL13-2 & Gorai.008G227100.1 & 3931 & 1176 & 391 & 4 & 40.457 & 9.13 & 8 \\
\hline GrAHL 14-1 & Gorai.007G280000.1 & 6475 & 1035 & 344 & 5 & 35.976 & 9.59 & 10.5 \\
\hline GrAHL14-2 & Gorai.007G345200.1 & 4396 & 1038 & 345 & 5 & 36.075 & 9.86 & 12 \\
\hline GrAHL14-3 & Gorai.013G186600.1 & 3669 & 1035 & 344 & 5 & 36.192 & 9.356 & 11.5 \\
\hline GrAHL-X1 & Gorai.002G160000.1 & 5307 & 1101 & 366 & 4 & 38.034 & 9.785 & 9 \\
\hline GrAHL-X2 & Gorai.006G158700.1 & 2517 & 1053 & 350 & 4 & 36.311 & 8.883 & 8.5 \\
\hline GrAHL-X3 & Gorai.009G408800.1 & 3520 & 993 & 330 & 4 & 33.683 & 8.456 & 4 \\
\hline GrAHL-X4 & Gorai.001G119100.1 & 2223 & 1095 & 364 & 4 & 38.243 & 7.851 & 4.5 \\
\hline GrAHL-X5 & Gorai.012G024700.1 & 8394 & 633 & 210 & 4 & 22.749 & 5.77 & -1.5 \\
\hline GrAHL15 & Gorai.011G267800.1 & 3780 & 933 & 310 & 0 & 32.515 & 5.898 & -4 \\
\hline GrAHL16-1 & Gorai.006G007800.1 & 771 & 771 & 256 & 0 & 27.271 & 9.111 & 7.5 \\
\hline GrAHL16-2 & Gorai.007G070000.1 & 1215 & 759 & 252 & 0 & 26.816 & 8.113 & 5 \\
\hline GrAHL17-1 & Gorai.001G133900.1 & 1721 & 894 & 297 & 0 & 30.26 & 9.235 & 10.5 \\
\hline GrAHL17-2 & Gorai.005G096700.1 & 921 & 921 & 306 & 0 & 31.725 & 7.472 & 5.5 \\
\hline GrAHL17-3 & Gorai.006G120100.1 & 684 & 684 & 227 & 0 & 24.17 & 7.234 & 3 \\
\hline GrAHL17-4 & Gorai.006G124100.1 & 684 & 684 & 227 & 0 & 24.232 & 6.944 & 2 \\
\hline GrAHL17-5 & Gorai.009G075100.1 & 1020 & 882 & 293 & 0 & 30.273 & 8.108 & 8.5 \\
\hline GrAHL17-6 & Gorai.009G230300.1 & 7250 & 957 & 318 & 0 & 33.38 & 8.214 & 11.5 \\
\hline GrAHL17-7 & Gorai.010G035300.1 & 1448 & 864 & 287 & 0 & 29.728 & 7.054 & 3 \\
\hline GrAHL17-8 & Gorai.013G253800.1 & 1304 & 987 & 328 & 0 & 33.21 & 10.682 & 10 \\
\hline GrAHL20-1 & Gorai.005G048000.1 & 1403 & 888 & 295 & 0 & 30.487 & 5.349 & -3.5 \\
\hline GrAHL20-2 & Gorai.006G247900.1 & 1008 & 852 & 283 & 0 & 28.921 & 5.62 & -3 \\
\hline GrAHL20-3 & Gorai.007G280400.1 & 909 & 909 & 302 & 0 & 30.368 & 5.954 & -2 \\
\hline GrAHL22-1 & Gorai.001G173500.1 & 870 & 870 & 289 & 0 & 30.852 & 7.738 & 4 \\
\hline GrAHL22-2 & Gorai.004G160700.1 & 1004 & 927 & 308 & 0 & 32.41 & 6.97 & 2 \\
\hline GrAHL22-3 & Gorai.007G091800.1 & 1768 & 903 & 300 & 0 & 31.472 & 6.512 & 0 \\
\hline GrAHL23-1 & Gorai.003G181200.1 & 804 & 804 & 267 & 0 & 27.75 & 6.856 & 2 \\
\hline GrAHL23-4 & Gorai.008G226900.1 & 1477 & 801 & 266 & 0 & 27.957 & 6.739 & 1.5 \\
\hline GrAHL23-2 & Gorai.004G185900.1 & 960 & 864 & 287 & 0 & 29.853 & 6.794 & 1.5 \\
\hline GrAHL23-3 & Gorai.006G216300.1 & 828 & 828 & 275 & 0 & 28.779 & 6.221 & -2 \\
\hline GrAHL24-1 & Gorai.003G167700.1 & 1374 & 930 & 309 & 0 & 33.106 & 6.798 & 2.5 \\
\hline
\end{tabular}


Table 1 Information of the AHL genes in G. raimondii (Continued)

\begin{tabular}{lllllllll}
\hline Gene name & Sequence ID & Gene (bp) & CDS (bp) & Protein(aa) & Intron & MW $^{\mathrm{a}}(\mathrm{kDa})$ & $\mathrm{pl}^{\mathrm{b}}$ & $\mathrm{Charge}$ \\
\hline GrAHL24-2 & Gorai.006G211500.1 & 900 & 900 & 299 & 0 & 31.775 & 6.704 & 1.5 \\
GrAHL24-3 & Gorai.008G240700.1 & 1751 & 924 & 307 & 0 & 32.791 & 6.584 & 0.5 \\
GrAHL25-1 & Gorai.005G215400.1 & 1776 & 846 & 281 & 0 & 28.239 & 9.008 & 4.5 \\
GrAHL25-2 & Gorai.012G138000.1 & 1800 & 846 & 281 & 0 & 28.419 & 8.982 & 5 \\
\hline
\end{tabular}

${ }^{\mathrm{a}}$ Molecular weight of the amino acid sequence, ${ }^{\mathrm{b}}$ Isoelectric point

tree was reconstructed by 1000 bootstrap replicates with $A H L$ proteins from $P$. patens $(P p), A$. thaliana $(A t), V$. vinifera $(V v), T$. cacao $(T c)$ and $G$. raimondii $(G r)$. The phylogenetic analysis showed that the AHLs were divided into two monophyletic clades, Clade-A and Clade-B, with 9 and 8 groups respectively (Fig. 1). Each group in Clade-A (except for AHL-X, no corresponding AHL gene in A. thaliana, named AHL-X) was composed of one $V v A H L$, one TcAHL, different number of $A t A H L$ and GrAHL respectively. Those groups in Clade-B were composed of various number of $A H L$ genes from $A$. thaliana (At), $V$. vinifera $(V v), T$. cacao (Tc) and $G$. raimondii (Gr). In $G$ raimondii, Clade-A contained 22 genes including the members of GrAHL1, GrAHL3, GrAHL5, GrAHL7, GrAHL9, GrAHL10, GrAHL13, GrAHL14, GrAHL-X1 and GrAHL-X2, while Clade-B contained 26 members including GrAHL15, GrAHL16, GrAH L17, GrAHL20, GrAHL22, GrAHL23, GrAHL24 and GrAH $L 25$. Each group of $G r A H L$ gene family usually contained 2-3 members, while the group of GrAHL17 had 8 members. The Group AHL15, AHL10 and AHL3 showed a more rigorous evolution pattern, with only one copy left in the genomes of the 4 examined species (Fig. 1, Additional file 3). This result indicated the different characteristics and the patterns of evolution in various group. The members of AHLs in G. raimondii, G.arboreum and G.hirsutum showed the preferably relationship of one-to-one correspondence except for AHL17 and AHL23, there were 4 AHL17 members in G. raimondii, 6 in G. arboreum, and 9 in G. hirsutum (Additional file 4). The AHLs from P. patens evolved into two clades, suggesting an expansion of the $A H L$ gene family in land plants posterior to the division between $P$. patens and the extant land plants [3].

\section{Conservation of gene structure and motifs among AHLs in cotton}

The AHL proteins were typically characterized by the presence of AT-hook motif(s) for binding DNA and PPC/ DUF296 domain for nuclear localization and interaction with each other or themselves [4]. To investigate the presence of homologous domain sequences and the degree of conservation in the two domains, AT-hook motif(s) and PPC domain, we performed multiple sequence alignment to generate sequence logos of the two domains in cotton against the MEME website (http://meme-suite.org/tools/ meme). 20 conserved motifs were predicted, and the specific amino acid sequences of each motif were also provided (Fig. 2, Additional file 8). Based on the number and composition of the AT-hook motif(s) and PPC/DUF 296 domain, AHL proteins were classified into three types (Type-I/-II/-III), with Type-I AHLs forming Clade-B, and the other two types together diversifying in Clade-A. Two types of AT-hook motifs ( $\mathrm{H} 1$ and $\mathrm{H} 2$ ) were found in the AHL proteins (Fig. 2). Both of $\mathrm{H} 1$ and $\mathrm{H} 2$ in the AHL proteins shared the same conserved R-G-R-P core, showing that the ability of bind the minor groove of AT-rich B-form DNA. The conservation of $\mathrm{H} 2$ with a longer core R-G-R-P$\mathrm{R}-\mathrm{K}-\mathrm{Y}$ heptapeptides was higher than that of the $\mathrm{H} 1$ in cotton. $\mathrm{H} 1$ was found only in Clade- $\mathrm{B}$, while $\mathrm{H} 2$ or $\mathrm{H} 1$ plus $\mathrm{H} 2$ were found in Clade-A (Fig. 2). The $A H L$ proteins in $T$. cacao, the closest related species of cotton, contained three types, while the AHL proteins in grape has only two types, Type-I and -III. The conserved structure of AHL9, AHL5 from 4 species contained 2 AT-hook motifs, indicating the distinct function in development. Almost all $A H L$ genes in cotton (except for Gorai.012G0247001, Ga04G1890.1, Gh D04G0182.1 and Gh_A05G3407.1, named AHL-X5) contained AT-hooking motif (s) and PPC/DUF296 domain. We considered $A H L-X 5$ s (Table 1, Additional files 1 and 2) in cotton as pseudogenes, because they contained the most regions of PPC/DUF296 domains although lacking the AThooking motifs and core sequences (motif 2), so these four genes were used as the members of $A H L$ family for further analysis.

To investigate the diversity of gene structure, we performed multiple sequence alignment to generate the exons/ intron pattern using the GSDS (http://gsds.cbi.pku.edu.cn/ chinese.php). The structures of $A H L$ genes can be divided into two types, with intronless and multiple-exon. The 26 $A H L$ genes in Clade-B showed intronless in Graimondii, while those in Clade-A with 5-6 exons (Fig. 2). Most of the AHL genes in both G. arboreum and G. hirsutum presented similar exon/intron gene structure. The exception was AHL20-2 in Clade-B, which had only one exon in G. raimondii, but its orthologous genes in both G. arboreum and G. hirsutum showed 4-5 introns in CDS, this indicating that the rapid evolution with intron-insertion (Additional file 5).

Chromosomal location and synteny analysis of AHL genes A total of $48 \mathrm{Gr} A H L$ genes were unevenly mapped onto 13 chromosomes of $G$. raimondii. Each chromosome 


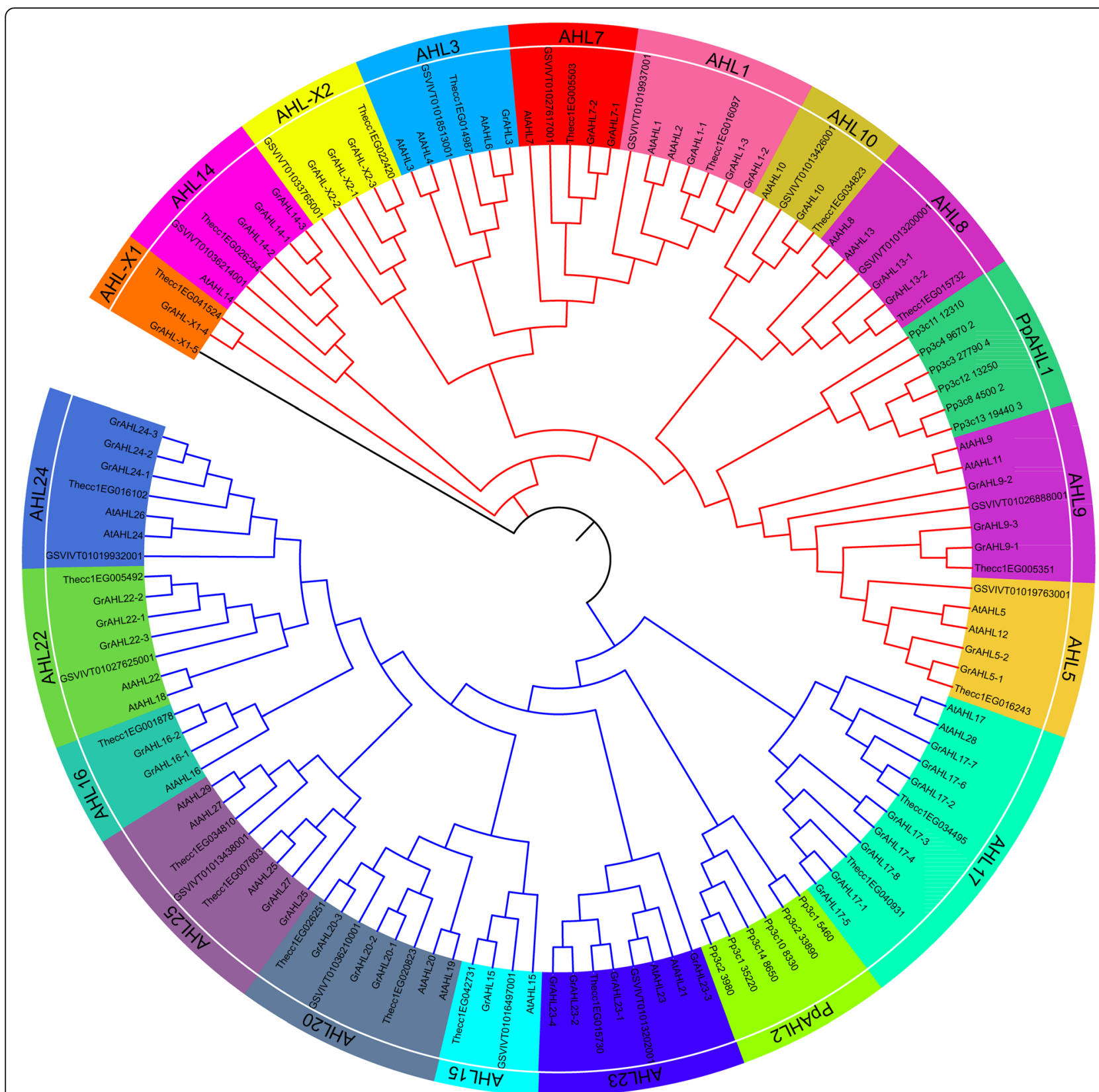

Fig. 1 Phylogenetic relationship among AHL proteins. The maximum-likelihood (ML) phylogenetic tree was constructed by MEGA 7.0 using 1000 bootstrap replicates for the AHL proteins from V. vinifera $(V V)$, A. thaliana $(A t), T$. cacao $(T c)$, G. raimondii $(G r)$ and P. patens (Pp). Clade-A indicated in blue branch lines and Clade-B in red branch lines. The black line showed the pesudogene

contained 3-6 AHL members usually. Chromosome 07 contained $8 A H L$ genes, while chromosome 10 and 11 had only one $A H L$, respectively (Fig. 3). The distribution of GaAHL and GhAHL genes showed similar to G. raimondii but some $A H L$ genes in scaffolds (Additional files 1 and 2).

We surveyed the collinear relationship among the orthologous $A H L$ genes from $V$. vinifera, T. cacao and G. raimondii to investigate the putative clues of evolutionary events. There were 15, 21, 29 and 48 AHL genes in $V$. vinifera, T. cacao, A. thaliana and G. raimondii, respectively. Specific loss and expansion of $A H L$ genes were found in four species. AHL16 and AHL17 were not found in $V$. vinifera, while $A H L-X$ not in $A$. thaliana. Most of $A H L$ genes showed one-to-one corresponding relationship in $V$. vinifera and $T$. cacao, while 2-4 orthologous genes were found in G. raimondii (Fig. 3, Additional file 3). In order to investigate the pattern of gene duplication, MCScanX was used to analyze $A H L$ gene family in G. raimondii, G. arboreum and G. hirsutum, the $A H L$ genes in G. raimondii and G. arboreum 

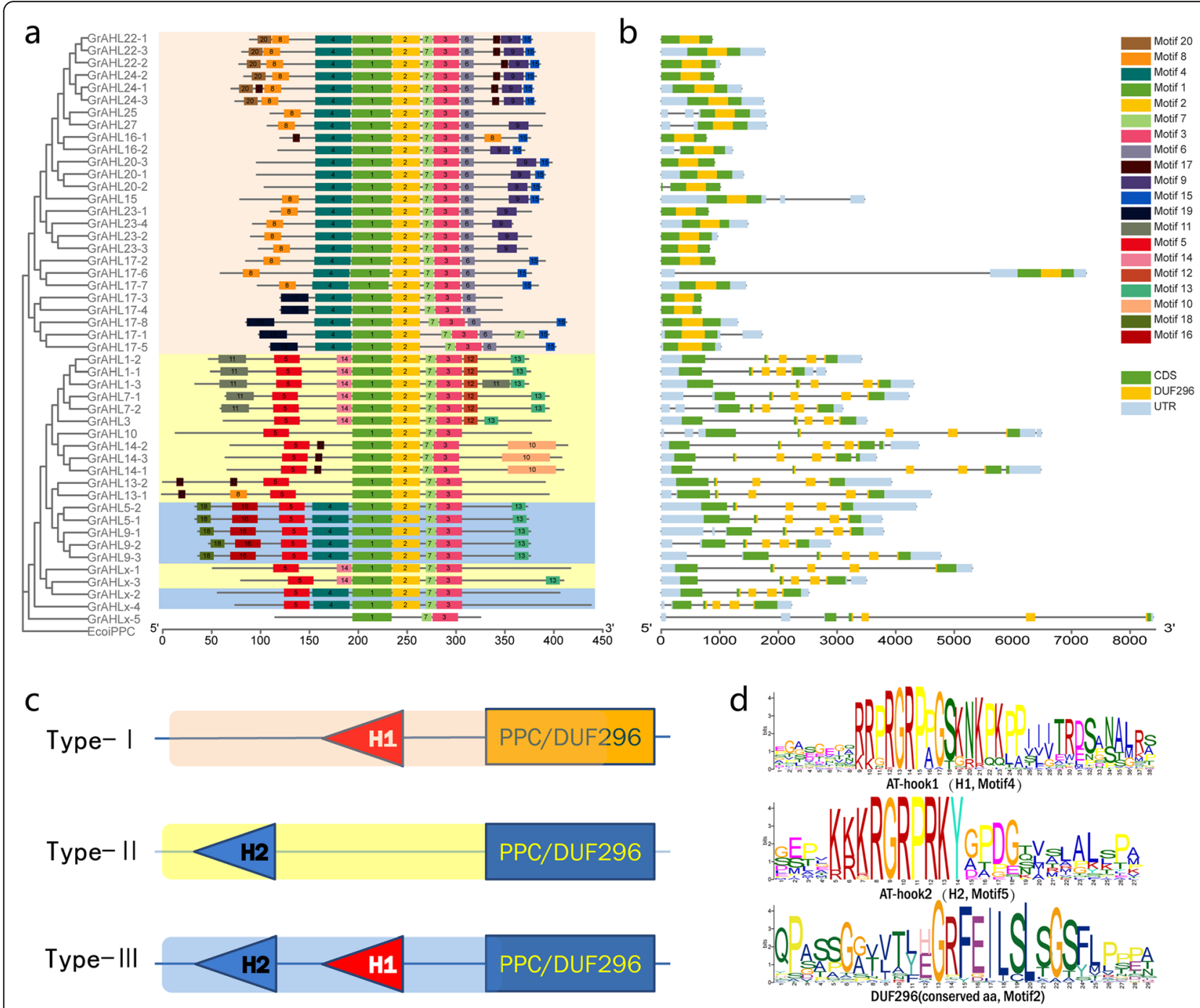

Fig. 2 The conserved motifs, Exon-intron structures of GrAHLS. a The maximum-likelihood phylogenetic tree was constructed by MEGA 7.0 using 1000 bootstrap replicates. Conserved motifs and gene structure were predicted from MEME and GSDS website (http://meme-suite.org/tools/meme, http://gsds.cbi.pku.edu.cn/chinese.php). The length of proteins and DNA sequence was estimated using the scale at the bottom. The motifs were displayed in the different colored boxes with various numbers, black line indicated the non-conserved amino acid or introns. $\mathbf{b}$ Light blue boxes indicate untranslated 5- and 3-regions; green boxes indicate exons. c The PPC domains were highlighted by yellow boxes. The topology of three types of AHL proteins in cotton based on the AT-hook motifs and PPC domain (motif 2). $\mathrm{H1}$ represented the AT-hook1 containing the conserved peptide in motif4; $\mathrm{H} 2$ represented the AT-hook2 containing the conserved peptide in motif5. $\mathbf{d}$ The conserved motifs analysis of sequence logo

showed the correspondent relationship between those from D-subgenome, A-subgenome in G.hirsutum respectively (Additional file 7). The result indicated that the expansion of GrAHL gene family were with segmental duplication or whole genome duplication (WGD), no tandem duplication were found (Fig. 4).

\section{Different evolution of $A H L$ genes in a and D subgenomes of G. hirsutum}

To explore the selective constrains among the orthologous AHL genes in G. raimondii, G. arboreum and G. hirsutum, we calculated $\mathrm{Ks}$, $\mathrm{Ka}$ and the $\mathrm{Ka} / \mathrm{Ks}$ ratio for the $A H L$ gene pairs (Additional files 9 and 10). It is generally believed that the value of Ks was not affected by natural selection, but that of Ka was affected by natural selection. The $\mathrm{Ka} / \mathrm{Ks}$ value can also explain positive selection $(\mathrm{Ka} / \mathrm{Ks}>1)$, neutral selection $(\mathrm{Ka} / \mathrm{Ks}=1)$ and negative selection $(\mathrm{Ka} / \mathrm{Ks}<1)$ during the evolution. In this study, 48 and 51 orthologous $A H L$ gene paris were identified by OrthoMCL between G. raimondii and Dsubgenome of G. hirsutum (GrAHL/GhAHL_Dt), and bewteen G. arboretum and A-subgenome of G. hirsutum (GaAHL/GhAHL_At), resprectively. The distributions of $\mathrm{Ka}$ and Ks between each pairs were shown in Fig. 5. The Ka of GrAHL/GhAHL_Dt ranged from 0.972745 to 1.08213, while Ks from 0.795064 to 1.08921 . The Ka of 


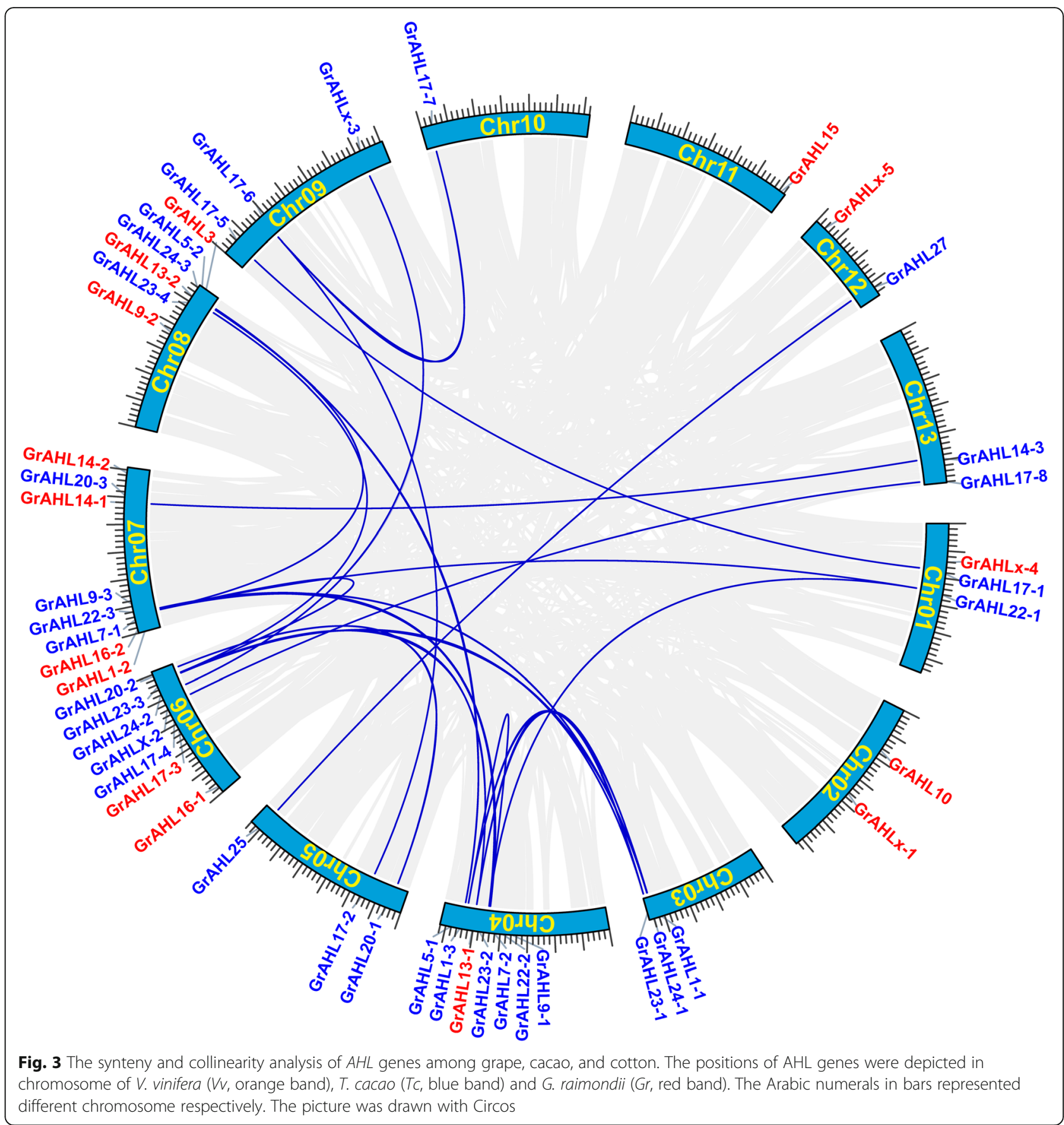

GaAHL/GhAHL_At ranged from 0.915553 to 1.03866, while Ks from 0.899268 to 1.30387 .19 gene pairs of GrAHL/GhAHL-Dt (39.6\%) with $\mathrm{Ka} / \mathrm{Ks}>1$ were subjected to positive selection, while 2 (AHL24-2 and AHL17-3) negative selection; 17 gene pairs of GaAHL/ GhAHL_At (33.3\%) with $\mathrm{Ka} / \mathrm{Ks}<1$ were subjected to negative selection, while only $A H L 17-8$ positive selection. The result suggested that the GhAHL genes derived from G. raimondii and G. arboreum underwent various selection directions during the evolution.

\section{Gene expression profiles of GhAHLs}

To explore the possible biological functions of $A H L s$, we inspected the expression patterns of different $A H L$ genes in G. hirsutum based on the RNA-seq data downloaded from CottonFGD (http://www.cottonfgd.com). The $A H L$ genes from $G$. hirsutum were expressed in different temporal and spatial patterns. Most GhAHL genes in CladeB (such as AHL20, AHL22, AHL23, AHL24) were found strongly up-regulated expression in the stem, but extremely lowly in fiber, ovule, leaf, petal, root, stamen and 

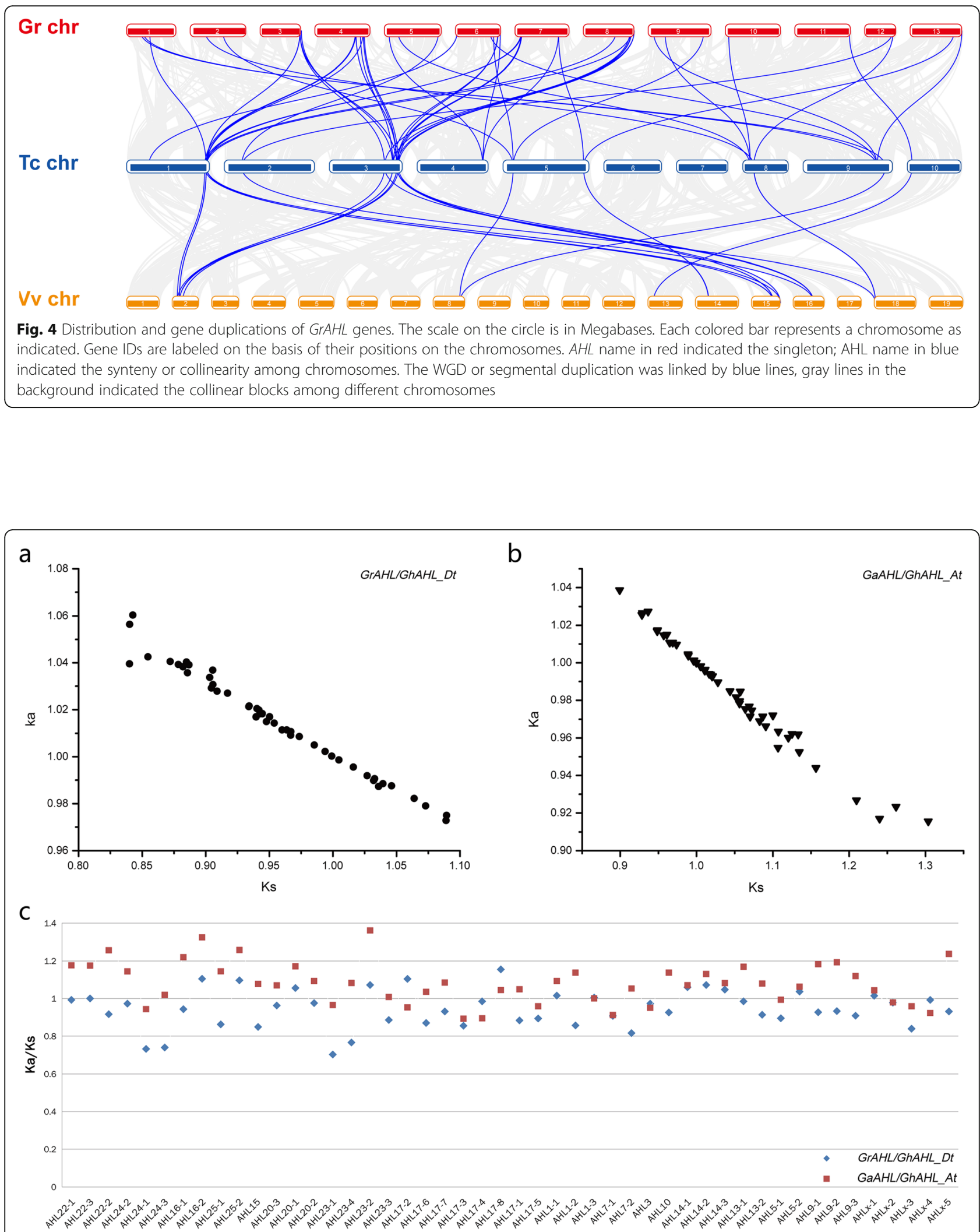

Fig. 5 The distribution of non-synonymous (Ka) and synonymous (Ks) nucleotide substitution values of and Ka/Ks ratio of orthologous pairs between GrAHL, GaAHL and GhAHL. a Ks analysis of GrAHL/GhAHL_Dt. b Ks analysis of GaAHL/GhAHL_At. c Ka/Ks analysisof GrAHL/GhAHL_Dt and GaAHL/GhAHL_At 
pistil. Some of GhAHL genes in Clade-A (such as, $A H L 1$, AHL7, AHL9 and AHL10) showed an extensive expression activity in different organs, highly expressed in the fiber and ovule, suggesting a special function of these genes in the development of cotton ovule (Fig. 6). Interestingly, two AHL20-2 genes inserted by introns in G. hirsutum showed higher expression activity in all organs and periods than other member in Clade- $B$. The expression of GrAHL showed similar pattern in different tissues (Additional file 6). The expression result showed that the AHLs within each clade shared similar expression patterns with each other; however, $A H L$ s in one monophyletic clade exhibited distinct expression patterns from those in the other clade.

For verification the data of RNA-seq, the qRT-PCR of six selected $A H L$ genes in G. hirsutum was performed to analyze the expression pattern in stem, root, leaf, flower and ovule $(-3,-1,0,1,3,5 \mathrm{DPA})$. The results showed that two $A H L$ genes (AHL22-1, AHL20-2) in Clade-B displayed higher expression in stem, and lower expression in leaf. Three $A H L$ genes (AHL9-1, AHL7-1and AHL10) in
Clade-A expressed highly in the early development of ovule. AHL16-1 expressed extremely lower in stem, root, leaf and flower (Fig. 7). The result coincided with the data of the RNA-seq, suggesting that the data from CottonFGD (http://www.cottonfgd.com) were reliable.

\section{Discussion}

Cotton is one of the most important economical crops worldwide, providing more than $90 \%$ of the natural fiber for textile industry. Previous research about the $A H L$ gene family has been performed in A. thaliana, P. patens and other monocot and dicot plants. In this study, we performed a comprehensive identification of $A H L$ genes in $G$. raimondii, G. arboreum, and G. hirsutum, with an aim of understanding the important and diverse roles of this gene family in regulation of growth and development in plants.

\section{Identification of AHL proteins}

In our study, 48, 51 and $99 A H L$ genes were identified from the G. raimondii, G. arboreum and G. hirsutum genomes, respectively. According to the phylogenetic
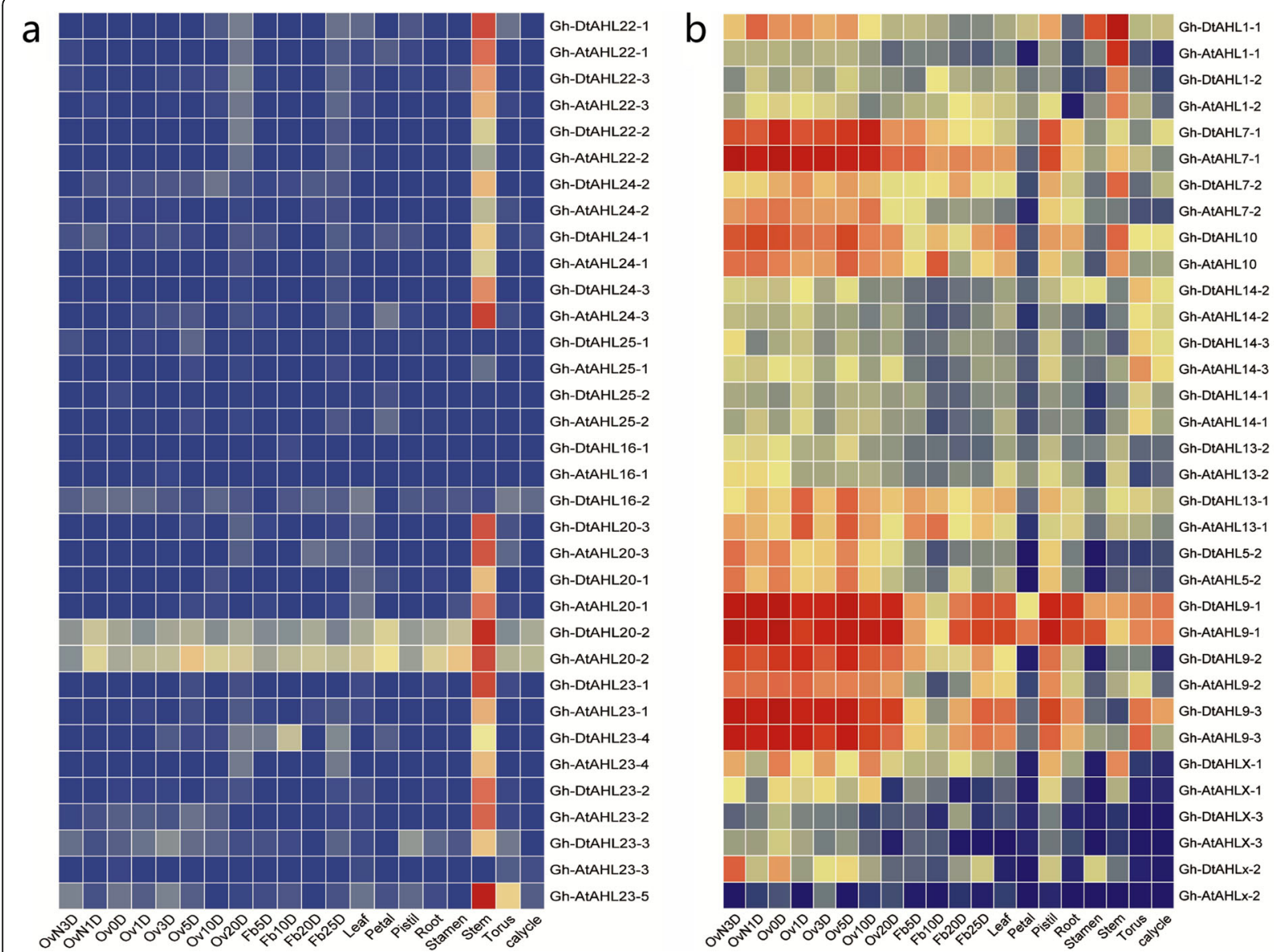

Fig. 6 The expression profiles of GhAHL genes. The heatmap was generated on the basis of RNA-seq data from the website (http://www.cottonfgd. com), the color scale was shown at the right of the figure. Higher expression levels were shown in red, and lower in blue. OvN3D, represented the ovule in 3rd days before anthesis, Ov0d, represented the ovule in 0 day of anthesis, fb5d, = represented the fiber in 5th day after anthesis(a, b) 

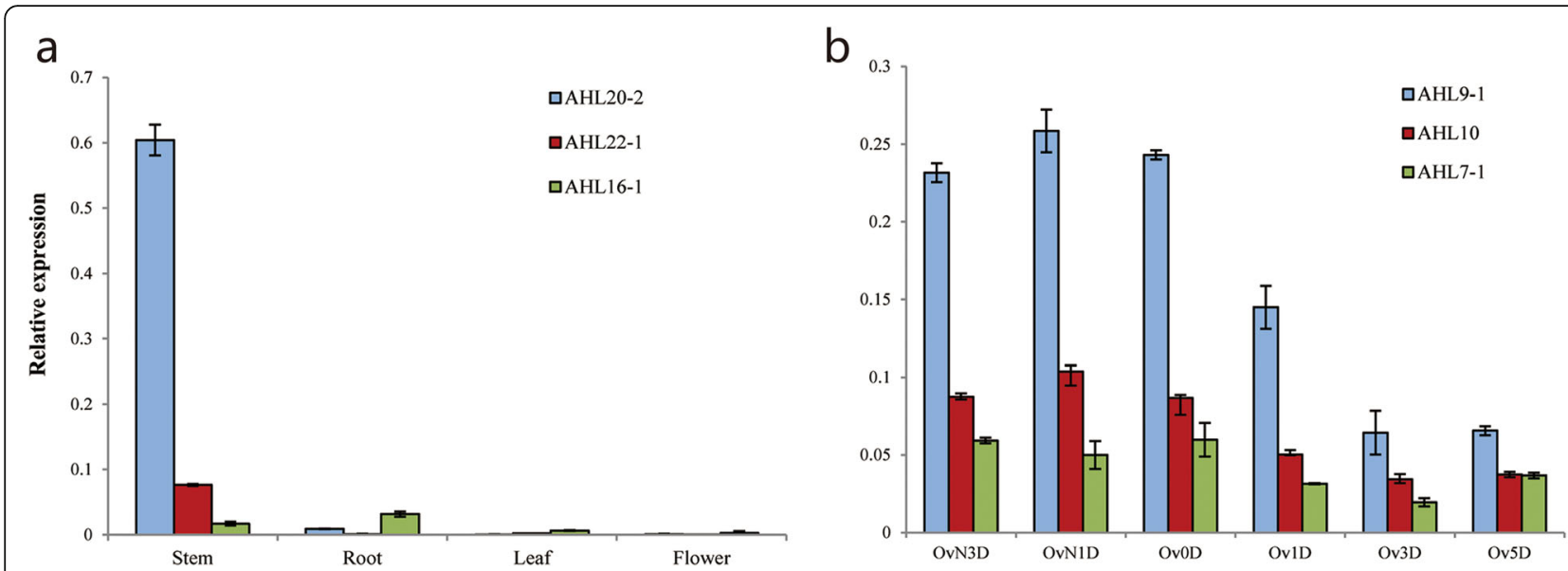

Fig. 7 The expression patterns of six AHL genes in G.hirsutum. qRT-PCR was conducted to analyze the relative expression of six AHL genes in stem, root, leaf, flower and ovule (-3,-1,0,1,3,5 DPA). a Expressed of AHL22-1, AHL20-2 and AHL16-1 genes in stem, root, leaf, flower. b Expressed of AHL9-1, AHL7-1 and AHL10 in - 3,-1,0,1,3,5DPA

analysis and gene structure, Ga07G1158.1 were regard as the member of the group of $A H L 9$, but noted as AHL1 in Version 2 of G. arboretum; Gh_D11G0864.1 should be regard as the member of AHL22, not AHL18 in notation. The group of $A H L-X 5$ (Gorai.012G0247001, Ga04G1890 Gh_D04G0182 and Gh_A05G3407) in 3 cotton species showed similar structure, containing the most regions of PPC/DUF296 domains, but the lack of the AT-hooking motifs, so we regarded these four genes as pseudogenes and the members of $A H L$ family for further analysis. GSVIVT01013438001 in grape containing AT-hooking motif, but lack of part sequences of PPC/ DUF296 domains, were also regard as the member of $A H L$ gene family, different to the study of Zhao et al. [1]. $12 A H L$ genes were obtained from P. patens, which differ from the $10 \mathrm{AHL}$ genes in the previous study maybe because of the annotation version of genome sequencing. Genomes of G. hirsutum are derived from hybridization between D-subgenome of $G$. raimondii and A-subgenome in G. arboreum [23-26]. The 47 of 48 GrAHL genes were located onto 13 chromosomes, showing one to one corresponding relationship with those of Dsubgenome in G. hirsutum. No member of GhAHL was located onto the Chromsome 06 in D-subgenome, while Gh Sca005047G03 was located on scaffolds. Based on the synteny analysis, we speculated that Gh_Sca005047G03 was likely located on Chromosome 06 of D-subgenome. GaAHL genes showed better linearity relationship to those in Asubgenome, it was speculated that Gh_Sca009301G01, Ga14 G0362, Ga14G0408 and Ga14G1507 were likely located on Gh_A11, GaChr09, GaChr06 and GaChr02, respectively (Additional file 8).

The $A H L$ genes are divided into Clade- $\mathrm{A}$ and Clade- $\mathrm{B}$, but the group members of Clade- $A$ and Clade- $B$ were respectively 5 and 4 in land plants [1], more than those from
$P$. patens, suggesting that an significant expansion of the $A H L$ gene family in land plants. $48 \mathrm{GrAHL}$ genes in G. raimondii were more than those from other species reported in previous report or closely-related species, such as T. cacao (21) and grape (15) [1]. Each group in Clade-A (except for AHL-X) was composed of one $V v A H L$, one TcAHL, different number of AtAHL and GrAHL, respectively. Most groups had 2-3 members in the diploid cotton, while the GrAHL17 had 8 members in G. raimondii, 9 in G. arboreum and $18 \mathrm{G}$. hirsutum, indicating a different expansion of the $A H L$ gene family. The synteny results showed that the expansion of $A H L$ family were with the WGD or segmental duplications, not tandem duplication. Related research suggested that the ancestor of Gossypium experienced a whole-genome duplication event after its divergence from $T$. coco ancestor $[23,24]$. So, we speculated that the numbers of the AHLs in G. raimondii or G. arboreum were more than that in $V$. vinifera and $T$. cacao maybe due to the specific WGD event in Gossypium ancestor after the divergence of cotton from T. cacao [23, 25]. The $A H L$ gene losses were also found in A. thaliana, group AHL- $X$ included the corresponding $A H L$ genes from G. raimondii, $V$. vinifera and $T$. cacao, no member were found in $A$. thaliana, suggesting that the different number of each group resulted from the various gene loss.

\section{Conservation of the $A H L$ gene family}

The $A H L$ gene family is a plant-specific family with conserved structure of AT-hook and PPC/DUF domain. The members of $A H L$ family present diversity not only in the sequence of AT-hook and PPC motifs, but also in gene length, gene structure, as well as in motif number. An analysis of sequence logo was performed for further investigating the divergence of AT-hook motif and the PPC domains in AHL proteins. AT-hook motif (s) could 
be distinguished by the phylogenetic relatedness of its homeodomains. Our results demonstrated that a longer core sequence R-G-R-P in $A H L$ proteins in cotton, especially in type II AT-hook motif, containing a more longer and conserved core R-G-R-P-R-K-Y heptapeptide. According to the AT-hook motif and PPC domain, the AHL proteins in cotton were divided into three types, agree with previous study [1, 3]. Two types of gene structure, with intronless and multiple-exon, were found in the $A H L$ genes of cotton. The $26 \mathrm{GrAHL}$ genes in Clade-B showed intronless, while those genes in Clade-A with 5-6 exons. The $A H L$ genes in $V$. vinifera presented another scenario, in which most of the $A H L$ genes contain multiple exons except for the sole-exon gene GSVIVT01027625. There were some exceptions in cotton and T. cacao, such as the inclusion of multiple exons in T. cacao Thecc1EG005492 and Thecc1EG034810, which were clustered in Clade-A. The difference of gene structure among the AHL2O-2 genes in different cotton species were showed in Fig. S4, GrAHL2O2 possessed only one exon while its orthologous members in G. arboreum and G. hirsutum contained multiple introns, suggesting a rapid evolutionary rate during the history of cotton. Furthermore, the $A H L$ genes in Clade-B in G. hirsutum were mainly specifically expressed in stem, with no detectable expression in other organs. Two members of AHL20-2 from A-subgenome and D-subgenome respectively, with multiple introns, expressed in various organs and tissues, suggesting that the gene structure may have some effects on gene expression pattern.

\section{Expression profile analysis of $\mathrm{AHL}$ in cotton}

The $A H L$ genes play important roles in plant development, floral transition and response to biotic and abiotic stress [1, 4, 10]. AHL20, AHL22, AHL23 and AHL24 were strongly up-regulated expressed in the stem, but extremely lowly in fiber, ovule, leaf, petal, root, stamen and pistil. AHL1, AHL7, AHL9 and AHL10 showed an extensive expression activity in different organs, highly expressed in the fiber and ovule, suggesting a special function of these genes in the development of cotton ovule. According to the phylogenetic analysis, Group of AHL3, AHL10 and AHL15 kept one copy left in $V$. vinifera, $T$. cacao, A. thaliana and $G$. raimondii, suggesting the more conserved function or vital roles in development. The gene expression patterns of GhAHL10 and GhAHL15 were observably different, GhAHL10 was observably expressed in all detected tissues and stages, while GhAHL15 were not detected expression in any detected tissues and stages. Compared with the homologous groups of $V$. vinifera and $T$. cacao, the members of $A H L 17$ were observably expanded to 8 in G. raimondii and 17 in G. hirsutum; no expression was detected in tissues and stages except AHL17-2 and AHL17-6, consistent with decreased gene expression levels after gene expansion in previous reports. The expression result showed that the AHLs within each clade shared similar expression patterns with each other; however, AHLs in one monophyletic clade exhibited distinct expression patterns from the ones in the other clade.

\section{Conclusions}

Previous studies have shown that the $A H L$ genes play important roles in plant growth and development, and response to biotic and abiotic stress. This study provides a comprehensive analysis of $A H L$ gene family in the genomes of three cotton species. All of the genes showed one-to-one homology relationship among three different genomes or subgenomes in cotton. Phylogenetic andSynteny analysis indicated that $A H L$ s in cotton were highly homologous to those in $V$. vinifera and $T$. cacao. $A H L$ genes are highly conserved among cotton and other plant species. Sequence analysis showed that segmental duplications were the major driving forces of $A H L$ family evolution, suggesting that $A H L s$ expanded with specific WGD in cotton. It is consistent with the identification and analysis results of the whole gene family of $A H L s$ in maize. The ratios of non-synonymous (Ka) and synonymous (Ks) substitution rates between orthologous gene pairs revealed that the $A H L$ genes of G.hirsutum had undergone through various selections during evolution, purifying selection mainly in A-subgenome and positive selection mainly in D-subgenome. A further expression analysis using RNAseq transcriptome and qRT-PCR indicated that most of AHLs of Clade-B expressed predominantly in stem, while those of Clade-A in ovules, suggesting that the AHLs within each clade shared similar expression patterns within each other, those genes might have experienced a functional divergence. Our study provided a reference for the further functional investigation of these selected candidate $A H L$ proteins.

\section{Methods \\ Identification of the $A H L$ genes}

To identify the $A H L$ gene family in cotton, the genome sequence and annotation data of four cotton species, including G. raimondii [23, 24], G. arboreum [25], G. hirsutum [26] and G. barbadense [11], were obtained from the CottonFGD (http://www.cottonfgd.org/) [27] by blastp against protein database and tblastn against genome databases using the query sequences of the 29 AHL proteins in $A$. thaliana acquired from TAIR 15 (http://www.arabidopsis. org), the E-value cut-off was set at 1.0e-5 to ensure confidence. The $A H L$ genes from $P$. patens $(P p), A$. thaliana $(A t), V$. vinifera $(V v), T$. cacao $(T c)$ were retrieved from the Phytozome database v12.1 (https://phytozome.jgi.doe.gov/ pz/portal.html). Redundant sequences were detected and deleted by manual. The candidate sequences were submitted to PROSITE for PPC domain (PS51742), those sequences comprised of AT-hook motif (s) and PPC domain 
were confirmed as $A H L$ genes for further analysis. Protein sequences of $A H L$ were submitted to ExPASy (http://web. expasy.org/protparam/) to predict the molecular weights (MW) and theoretical isoelectric points (pI) and charge.

\section{Chromosomal location and collinearity analysis}

The information of the AHLs loci on chromosome was obtained from annotation gff3 files. The Gene Structure Display Server (http://gsds.cbi.pku.edu.cn/) was used for gene structure analysis. Conserved protein motifs of the AHLs were predicted by the MEME program (http:// meme-suite.org/tools/meme). The parameters of MEME were optimum width, 3-50; number of repetitions, any; maximum number of motifs, 20. A schematic diagram of gene structure was redrawn by Circos. The MCscanX program was used to identify GrAHL duplications as previous described by Wang et al. [28]. Total 37,505 proteins sequences were used by all-all BLAST with evalue $<1.0 \mathrm{e}-5$. All genes were classified into various types of duplications, dispersed, singleton, WGD or segmental and tandem duplications. A schematic diagram of the putative WGD or segmental duplications of GrAHL was constructed using the Circos, and the AHLs with WGD or segmental duplications were linked by lines.

\section{Phylogenetic analysis and classification of $A H L$ genes in cotton}

For phylogenetic analysis, All AHL amino sequences from $P$. patens $(P p), A$. thaliana $(A t), V$. vinifera $(V v), T$. cacao $(T c)$ and three cotton species were aligned by ClustalX v1.83 with default parameters [29]. MEGA 7.0 was used to find best model and construct the Maximum likelihood (ML) tree with bootstrap test of 1000 replicates, the model of JTT $+\mathrm{G}$ was selected as the best model. Neighbor-Joining (NJ) phylogenetic trees were also generated in MEGA 7.0 to validate the ML phylogenetic trees [30].

\section{Calculation of $\mathrm{Ka} / \mathrm{Ks}$ of $A H L$ genes in cotton}

The orthologs of the AHL genes in G. raimondii, G. arboreum and G. hirsutum were identified by OrthoMCL [31]. The orthologous gene pairs of AHLs were aligned by codons with Muscle in MEGA 7.0 software. Non-synonymous (Ka) and synonymous (Ks) substitution rates and $\mathrm{Ka} / \mathrm{Ks}$ ratios of were determined by the model average (MA) and model (MS) in Kaks_Calculator 2.0 program [32], respectively.

\section{Expression profiles of GhAHL genes}

For analyzing the expression profile of GhAHL and GrAHL genes in different tissues and development stages, the expression data of fragments-per-kilobase-per-million (FPKM) were retrieved from the genome-wide RNA-seq dataset in CottonFGD (http://www.cottonfgd.com/data) and CCnet website (http://structuralbiology.cau.edu.cn/ gossypium), respectively. For each RNA-seq analysis, transcripts were assembled using Cufflinks software [33]. The heatmap charts were drawn according to gene expression values (FPKM).

\section{Quantitative RT-PCR (qRT-PCR) for GhAHL genes}

The upland cotton (TM-1) seeds were germinated on a wet germinated disc for 3 days at $28^{\circ} \mathrm{C}$, and then transferred to a liquid culture medium. Total RNA was extracted from the seedlings. The leaves, root and stem were collected and were immediately frozen in liquid nitrogen for RNA extraction. Blossom in full bloom, and then take the first 3 days, 1 day, 0,1 days after flowering, flowering after 3 days, 5 days after flowering ovule and flower liquid nitrogen treatment $-80^{\circ} \mathrm{C}$ after preservation; Total RNA was extracted from the seedlings. cDNA was synthesized by using an EASYspin Plus Plant RNA Kit (Aidlab) with gDNA Eraser (Takara). The qRT-PCR reactions were conducted using a SYBR Green I Master mixture (Roche, Basel, Switzerland) according to the manufacturer's protocol on a Light Cycler 480II system (Roche, Switzerland). Cotton ACTIN14 (GenBank accession number: AY305733) was used as an internal control in the PCR assays. The primers designed for qRT-PCR were showed in Additional file 11. The qRT-PCR was completed with three biological replicates, each comprising three technical replicates. The PCR conditions were as follows: $95^{\circ} \mathrm{C}$ for $30 \mathrm{~s} ; 40$ cycles of $95^{\circ} \mathrm{C}$ for $5 \mathrm{~s}, 60^{\circ} \mathrm{C}$ for $1 \mathrm{~min}$, and $72{ }^{\circ} \mathrm{C}$ for $10 \mathrm{~s} ; 50{ }^{\circ} \mathrm{C}$ for $30 \mathrm{~s}$. The relative gene expression levels were calculated based on the $2^{-\Delta \Delta C \mathrm{~T}}$ method [34].

\section{Supplementary information}

Supplementary information accompanies this paper at https://doi.org/10. 1186/s12864-019-6406-6.

Additional file 1. - Information of AHLs in G. arboretum. a Molecular weight of the amino acid sequence, $b$ Isoelectric point

Additional file 2. - Information of AHLs in G. hirsutum. a Molecular weight of the amino acid sequence, $b$ Isoelectric point

Additional file 3. - The orthologous relationship and type of AHL proteins in V. vinifera, T. cacao, A.thaliana and G. raimondii. The forms in pink indicated the Type-I AHL genes, those in yellow indicated the TypeIII AHL genes and those in blue indicated the Type-II AHL genes. The lines repented the loss of orthologous gene

Additional file 4. - Phylogenetic relationship of $A H L$ proteins in cotton. AHL proteins from G. raimondii, G. arboreum and G. hirsutum are marked with blue rhombus, green squares, and red rhombus squares, respectively

Additional file 5. - The variations of gene structures and motifs of AHL20-2 from G. raimondii, G. arboreum and G. hirsutum. Gene structure and conserved motifs were predicted from the GSDS and MEME website. The length of proteins and DNA sequence was estimated using the scale at the bottom. The motifs were displayed in different colored boxes with Arabic numerals; black line indicated the non-conserved amino acid or intron. Gray boxes indicate untranslated 5- and 3-regions, blue boxes indicate exons. The sequences of motifs were listed in additional file 6

Additional file 6. - The sequences of motifs predicted by MEME (http:// meme-suite.org/tools/meme) 
Additional file 7. - The expression profiles of GrAHLS. The heatmap was generated on the basis of RNA-seq data from the website (http://structur albiology.cau.edu.cn/gossypium), the color scale was shown at the right. Higher expression levels were shown in red, and lower in blue. DPA represented the day of ovule after anthesis

Additional file 8. - The circos map of AHLs in G. raimondii, G. arbretum and $G$. hirsutum. The collinearity of AHL genes between G. raimondii and $D$-subgenome in $G$. hirsutum were showed in orange lines, that between G. arbretum and the A-subgenome in G. hirsutum in blue lines. AHL genes located in scaffolds were showed in red lines, and the locations of scaffolds were putatived

Additional file 9. - Ka, Ks and Ka/Ks ratio between orthologous genes pairs from $G$. raimondii and D-subgenome in $G$. hirsutum

Additional file 10. $-\mathrm{Ka}$, Ks and $\mathrm{Ka} / \mathrm{Ks}$ ratio between orthologous gene pairs from $G$. arbretum and A-subgenome of $G$. hirsutum

Additional file 11. - The primers designed for QRT-PCR

\section{Abbreviations}

AHL: AT-Hook Motif Containing Nuclear Localized; BLAST: Basic Local Alignment Search Tool; DPA: Days post anthesis; FPKM: Fragments per kilobase of transcript per million mapped fragments; G. arboreum: Gossypium arboreum; G. hirsutum: Gossypium hirsutum; G. raimondii: Gossypium raimondii; GIK: GIANT KILLER; Ka: Non-synonymous; Ks: Synonymous; ML: Maximum likelihood; MW: Molecular weight; NJ: Neighbour Joining; $P$. patens: Physcomitrella patens; pl: Isoelectric point; qRT-PCR: Quantitative realtime polymerase chain reaction; T. cacao : Theobroma cacao; V. vinifera : Vitis vinifera; WGD: Whole genome duplication

\section{Acknowledgements}

Not applicable

\section{Authors' contributions}

Z.-Y.S. planned and designed the research. Z.-L.J. wrote the manuscript. L. Y.J, C. W, Y.-J.B, L.Y, L.-Q.L., P.-J.W, F.-S.T performed the experiments. S.J. supervised the research. Z.-L.J and L.-Y.J. contributed equally. All authors read and approved the final manuscript.

\section{Funding}

The study was supported in part by National Science Foundation in China (31871680), the project of state key lab of cotton biology (CB2017C12).

\section{Availability of data and materials}

All another data generated or analyzed during this study are included in this published article and its Additional files.

\section{Ethics approval and consent to participate}

Not applicable.

\section{Consent for publication}

Not applicable.

\section{Competing interests}

The authors declare that they have no competing interests.

\section{Author details}

${ }^{1}$ State Key Laboratory of Cotton Biology, Institute of Cotton Research of CAAS, Anyang 455000, Henan, China. ${ }^{2}$ Research Base, Anyang Institute of Technology, State Key Laboratory of Cotton Biology, Anyang 455000, Henan, China. ${ }^{3}$ Shihezi University, Shihezi 832003, Xinjiang, China.

\section{Received: 31 May 2019 Accepted: 16 December 2019}

\section{Published online: 22 January 2020}

\section{References}

1. Zhao J, Favero DS, Qiu J, Roalson EH, Neff MM. Insights into the evolution and diversification of the AT-hook motif nuclear Localized gene family in land plants. BMC Plant Biol. 2014;14(1):266.

2. Aravind L, Landsman D. AT-hook motifs identified in a wide variety of DNAbinding proteins. Nucleic Acids Res. 1998;26(19):4413-21.
3. Huth JR, Bewley CA, Nissen MS, Evans JN, Reeves R, Gronenborn AM, Clore GM. The solution structure of an HMG-I (Y)-DNA complex defines a new architectural minor groove binding motif. Nat Struct Biol Mol Biol. 1997;4(8):657-65.

4. Zhao J, Favero DS, Peng H, Neff MM. Arabidopsis thaliana AHL family modulates hypocotyl growth redundantly by interacting with each other via the PPC/DUF296 domain. Proc Natl Acad Sci U S A. 2013;110(48):4688-97.

5. Favero DS, Jacques CN, Iwase A, Le KN, Zhao J, Sugimoto K, Neff MM. SUPPRESSOR OF PHYTOCHROME B4-\#3 represses genes associated with auxin signaling to modulate hypocotyl growth. Plant Physiol. 2016;171(4): 2701-16.

6. Street IH, Shah PK, Smith AM, Avery N, Neff MM. The AT-hook-containing proteins SOB3/AHL29 and ESC/AHL27 are negative modulators of hypocotyl growth in Arabidopsis. Plant J. 2008:54(1):1-14.

7. Xiao C, Chen F, Yu X, Lin C, Fu YF. Over-expression of an AT-hook gene, AHL22, delays flowering and inhibits the elongation of the hypocotyl in Arabidopsis thaliana. Plant Mol Biol. 2009;71(1-2):39-50.

8. Gallavotti A, Malcomber S, Gaines C, Stanfield S, Whipple C, Kellogg E, Schmidt RJ. BARREN STALK FASTIGIATE1 is an AT-hook protein required for the formation of maize ears. Plant Cell. 2011;23(5):1756-71.

9. Jia QS, Zhu J, Xu XF, Lou Y, Zhang ZL, Zhang ZP, Yang ZN. Arabidopsis AThook protein TEK positively regulates the expression of arabinogalactan proteins for Nexine formation. Mol Plant. 2015;8(2):251-60.

10. Jin Y, Luo Q, Tong H, Wang A, Cheng Z, Tang J, Li D, Zhao X, Li X, Wan J, et al. An AT-hook gene is required for Palea formation and floral organ number control in rice. Dev Biol. 2011;359(2):277-88.

11. Liu X, Zhao B, Zheng HJ, Hu Y, Lu G, Yang CQ, Chen JD, Chen JJ, Chen DY, Zhang $L$, et al. Gossypium barbadense genome sequence provides insight into the evolution of extra-long staple fiber and specialized metabolites. Sci Rep. 2015;5(1):14139

12. Xu Y, Wang Y, Stroud H, Gu X, Sun B, Gan ES, Ng KH, Jacobsen SE, He Y, Ito T. A matrix protein silences transposons and repeats through interaction with retinoblastoma-associated proteins. Curr Biol. 2013;23(4):345-50.

13. $\mathrm{Ng} \mathrm{KH}$, Ito T. Shedding light on the role of AT-hook/PPC domain protein in Arabidopsis thaliana. Plant Signal Behav. 2010;5(2):200-1.

14. Ng KH, Yu H, Ito T. AGAMOUS controls GIANT KILLER, a multifunctional chromatin modifier in reproductive organ patterning and differentiation. PLoS Biol. 2009; 7(11):e1000251.

15. Yun J, Kim YS, Jung JH, Seo PJ, Park CM. The AT-hook motif-containing protein AHL22 regulates flowering initiation by modifying FLOWERING LOCUS T chromatin in Arabidopsis. J Biol Chem. 2012;287(19):15307-16.

16. Kim SY, Kim YC, Seong ES, Lee YH, Park JM, Choi D. The chili pepper CaATL1: an AT-hook motif-containing transcription factor implicated in defence responses against pathogens. Mol Plant Pathol. 2007:8(6):761-71.

17. Lu H, Zou Y. Feng N (2010) overexpression of AHL20 negatively regulates defenses in Arabidopsis. J Integr Plant Biol. 2010;52(9):801-8.

18. Matsushita A, Furumoto T, Ishida S, Takahashi Y. AGF1, an AT-hook protein, is necessary for the negative feedback of AtGA3ox1 encoding GA 3-oxidase. Plant Physiol. 2007;143(3):1152-62.

19. Zhou L, Liu Z, Liu Y, Kong D, Li T, Yu S, Mei H, Xu X, Liu H, Chen L. A novel gene OSAHL1 improves both drought avoidance and drought tolerance in rice. Sci Rep. 2016:6(1):30264.

20. Kim HB, Oh CJ, Park YC, et al. Comprehensive analysis of AHL homologous genes encoding AT-hook motif nuclear Localized protein in rice. J Biochem Mol Biol. 2011;44(10):680-5.

21. Bishop EH, Kumar R, Luo F, Saski C, Sekhon RS. Genome-wide identification, expression profiling, and network analysis of AT-hook gene family in maize. Genomics. 2019.

22. Delaney SK, Orford SJ, Martinharris M, Timmis JN. The fiber specificity of the cotton FSItp4 gene promoter is regulated by an AT-rich promoter region and the AT-hook transcription factor GhAT1. Plant Cell Physiol. 2007;48(10): 1426-37.

23. Wang K, Wang Z, Li F, Ye W, Wang J, Song G, Yue Z, Cong L, Shang H, Zhu $S$, et al. The draft genome of a diploid cotton Gossypium raimondii. Nat Genet. 2012:44(10):1098-103.

24. Paterson AH, Wendel JF, Gundlach $H$, Guo H, Jenkins J, Jin D, Llewellyn $D$, Showmaker KC, Shu S, Udall J. Repeated polyploidization of Gossypium genomes and the evolution of spinnable cotton fibres. Nature. 2012; 492(7429):423-7.

25. Li F, Fan G, Wang K, Sun F, Yuan Y, Song G, Li Q, Ma Z, Lu C, Zou C, et al. Genome sequence of the cultivated cotton Gossypium arboreum. Nat Genet. 2014;46(6):567-72 
26. Li F, Fan G, Lu C, Xiao G, Zou C, Kohel RJ, Ma Z, Shang H, Ma X, Wu J, et al. Genome sequence of cultivated upland cotton (Gossypium hirsutum TM-1) provides insights into genome evolution. Nat Biotechnol. 2015;33(5):524-30.

27. Zhu T, Liang C, Meng Z, Sun G, Meng Z, Guo S, Zhang R. CottonFGD: an integrated functional genomics database for cotton. BMC Plant Biol. 2017; 17(1):101.

28. Wang Y, Tang H, DeBarry JD, Tan X, Li J, Wang X, Lee TH, Jin H, Marler B, Guo H. MCScanX: a toolkit for detection and evolutionary analysis of gene synteny and collinearity. Nucleic Acids Res. 2012;40(7):e49.

29. Larkin MA, Blackshields G, Brown NP, Chenna R, McGettigan PA, McWilliam $H$, Valentin F, Wallace IM, Wilm A, Lopez R, et al. Clustal W and Clustal X version 2.0. Bioinformatics. 2007;23(21):2947-8.

30. Kumar S, Stecher G, Tamura K. MEGA7: molecular evolutionary genetics analysis version 7.0 for bigger datasets. Mol Biol Evol. 2016;33(7):1870-4.

31. Li L, Stoeckert CJ, Roos DS. OrthoMCL: identification of ortholog groups for eukaryotic genomes. Genome Res. 2003;13(9):2178-89.

32. Wang D, Zhang Y, Zhang Z, Zhu J, Jun Y. KaKs_Calculator 2.0: A toolkit incorporating gamma-series methods and sliding window strategies. Genomics Proteomics Bioinfo. 2010;8(1):77-80.

33. Trapnell C, Roberts A, Goff O, Pertea G, Kim D, Kelley DR, Pimentel H, Salzberg SL, Rinn JL, Pachter L. Differential gene and transcript expression analysis of RNA-seq experiments with TopHat and cufflinks. Nat Protoc. 2013;7(3):562-78.

34. Schmittgen TD, Livak KJ. Analyzing real-time PCR data by the comparative $c(t)$ method. Nat Protoc. 2008;3(6):1101-8.

\section{Publisher's Note}

Springer Nature remains neutral with regard to jurisdictional claims in published maps and institutional affiliations.

Ready to submit your research? Choose BMC and benefit from:

- fast, convenient online submission

- thorough peer review by experienced researchers in your field

- rapid publication on acceptance

- support for research data, including large and complex data types

- gold Open Access which fosters wider collaboration and increased citations

- maximum visibility for your research: over $100 \mathrm{M}$ website views per year

At BMC, research is always in progress.

Learn more biomedcentral.com/submissions 